\title{
Possible Predictive Factors for Low Back and Pelvic Pain at Three Months Postpartum Assessed in a Prospective Study from Early Pregnancy until the Postpartum Period
}

\author{
Yuko Uemura ${ }^{*}$, Toshiyuki Yasui ${ }^{2}$, Kimiyo Horike ${ }^{3}$, Kazuhisa Maeda ${ }^{3}$, Hirokazu Uemura4, \\ Mari Haku, Reiko Sakae', Keiko Matsumura1 \\ ${ }^{1}$ Department of Nursing, Kagawa Prefectural University of Health Sciences, Kagawa, Japan \\ ${ }^{2}$ Department of Reproductive and Menopausal Medicine, Institute of Biomedical Sciences, Tokushima University Graduate \\ School, Tokushima, Japan \\ ${ }^{3}$ Shikoku Medical Center for Children and Adults, Kagawa, Japan \\ ${ }^{4}$ Department of Preventive Medicine, Institute of Biomedical Sciences, Tokushima University Graduate School, \\ Tokushima, Japan \\ ${ }^{5}$ Department of Midwifery, Institute of Biomedical Sciences, Tokushima University Graduate School, Tokushima, Japan \\ Email:*uemura@chs.pref.kagawa.jp
}

How to cite this paper: Uemura, Y., Yasui, T., Horike, K., Maeda, K., Uemura, H., Haku, M., Sakae, R. and Matsumura, K. (2018) Possible Predictive Factors for Low Back and Pelvic Pain at Three Months Postpartum Assessed in a Prospective Study from Early Pregnancy until the Postpartum Period. Open Journal of Nursing, 8, 552-566.

https://doi.org/10.4236/ojn.2018.88041

Received: August 1, 2018

Accepted: August 25, 2018

Published: August 28, 2018

Copyright $\odot 2018$ by authors and Scientific Research Publishing Inc. This work is licensed under the Creative Commons Attribution International License (CC BY 4.0).

http://creativecommons.org/licenses/by/4.0/

(c) (i) Open Access

\begin{abstract}
Background: Low back and pelvic pain (LBPP) is an important issue related to mental health as well as to difficulty for daily life activity during early pregnancy and in the postpartum period. We examined the intensity of LBPP, difficulty for daily life activity and mental health and their associations from early pregnancy until 3 months postpartum in a prospective study. We also examined predictive factors for LBPP at 3 months postpartum. Methods: We recruited 55 pregnant women who responded to questionnaires in the first, second and third trimesters and at one week, one month and 3 months postpartum. We designed a self-administered questionnaire including a visual analog scale (VAS), pregnancy mobility index (PMI) of LBPP and Edinburgh postnatal depression scale (EPDS). Results: There were significant differences in the proportions of women with LBPP in the first trimester (63.6\%), second trimester $(78.2 \%)$ and third trimester $(83.6 \%)$ and at one week postpartum (80.0\%), one month postpartum (69.1\%) and 3 months postpartum (56.4\%). VAS score at 3 months postpartum was significantly correlated with VAS scores at the first trimester $(\mathrm{r}=0.410, \mathrm{p}=0.002)$, second trimester $(\mathrm{r}=0.298$, $\mathrm{p}=0.027)$ and one month postpartum $(\mathrm{r}=0.476, \mathrm{p}<0.001)$. VAS score at 3 months postpartum was significantly correlated with PMI scores at the first trimester and one month postpartum. The presence of LBPP at 3 months
\end{abstract}


postpartum was significantly associated with the proportion of women with LBPP at the first trimester. In multiparous women, predictive factor of LBPP at 3 months postpartum was a past history of LBPP in the previous pregnancy. Conclusion: The proportion of women with LBPP and the intensity of LBPP were increased at late pregnancy and gradually decreased until 3 months postpartum. LBPP at 3 months postpartum was associated with LBPP at the first trimester and a past history of LBPP in a previous pregnancy was an important factor for prediction of LBPP at 3 months postpartum. Provision of information regarding LBPP and confirmation of LBPP before pregnancy in addition to assessment of LBPP at early pregnancy are necessary for reduction of LBPP at 3 months postpartum.

\section{Keywords}

Low Back Pain, Pelvic Pain, Pregnancy, Prospective Study

\section{Introduction}

Many studies have shown that pregnancy-related low back and pelvic pain (LBPP) occurs from the second or third trimester to postpartum [1] [2] [3] [4]. Given that $65.6 \%$ of women in early pregnancy complained of LBPP [5] and that $40 \%$ of women at 6 months postpartum complained of LBPP [6] [7], LBPP is an important issue that affects pregnant women for a long period. In addition, continuation of LBPP has effects on infant rearing and return to work. Reduction of the severity of LBPP may improve the quality of daily life during pregnancy and postpartum since severe LBPP causes difficulty in daily life activity during the second trimester and during the first month postpartum [8]. However, there has been no study on the association between intensity of LBPP and difficulty in daily life activity for a long period from early pregnancy to 3 months postpartum.

Physical symptoms including pain are closely related to mental health during early pregnancy and the postpartum period. It has been reported that women who had experienced a greater number of physical health problems had an increased risk of depressive symptoms in early pregnancy [9]. It has also been reported that physical health problems such as tiredness and back pain in the early postnatal period were associated with poor mental health throughout the first 12 months postpartum [10]. Gutke et al. [11] reported that postpartum depressive symptoms were 3 times more prevalent in women having lumbopelvic pain than in women without lumbopelvic pain. However, there has been no prospective study on the association of LBPP during pregnancy with mental health at 3 months postpartum.

Postpartum women have motions for infant rearing other than daily life motions, although the change in the shape of the body during pregnancy gradually returns to the shape before pregnancy. Mukkannavar et al. [12] reported that a 
sitting position during breast-feeding was significantly associated with increased risk of pelvic girdle pain. Postpartum women can comfortably rear an infant if they do not have LBPP. Given that LBPP at 3 months postpartum influences not only daily life activity but also infant rearing and return to work, it is important to predict LBPP early after delivery. In Japan, postpartum women receive regular medical examinations from early pregnancy to 1 month postpartum but do not have regular medical examinations thereafter. If medical staff knew the best time for taking action to prevent LBPP, they may be able to take early actions for management and prevention of LBPP. At the early stage of pregnancy, prediction of LBPP at 3 months postpartum would be useful. However, there has been no prospective study on assessment of LBPP in the prospective study during early pregnancy and 3 months postpartum. In addition, the associations of difficulty for daily life activity and mental health during pregnancy with intensity of LBPP at 3 months postpartum have not been reported. We therefore examined the intensity of LBPP, difficulty for daily life activity and mental health from early pregnancy until 3 months postpartum in a prospective study and we examined the associations of LBPP at early pregnancy with LBPP-related factors thereafter. We also examined predictive factors for LBPP at 3 months postpartum.

\section{Methods}

\subsection{Subjects and Methods}

The study protocol was reviewed and approved by the Ethics Review Board of Tokushima University Hospital (approval no. 2201). The study was conducted from March 2015 to August 2017 in a birth center in Kagawa Prefecture in Japan. The number of hospital bed is 689 and the number of delivery is about 500 per year in the birth center. We distributed questionnaires regarding LBPP to 300 pregnant women at the first trimester who had undergone a prenatal health checkup at an obstetrics and gynecology clinic. Women with orthopedic disorders or neurological diseases that affected their activities regardless of pregnancy were excluded. Pregnant women having high risks such as twin pregnancy, severe infectious disease and mental disorders were also excluded. Participants were informed of the purposes and procedure of the study. We asked women who gave consent for research cooperation to respond to questionnaires at the first, second and third trimesters and at one week, one month and three months postpartum. The numbers of collected questionnaires were 231 in the first trimester (mean period: 14.8 weeks; 10 - 19 weeks), 171 in the second trimester (mean period: 25.8 weeks; 25 - 28 weeks), 151 in the third trimester (mean period: 35.7 weeks; 35 - 38 weeks), 89 at one week postpartum, 80 at one month postpartum and 55 at 3 months postpartum. Fifty-five pregnant women who responded to questionnaires in all six stages were used as subjects for this study (Figure 1). 


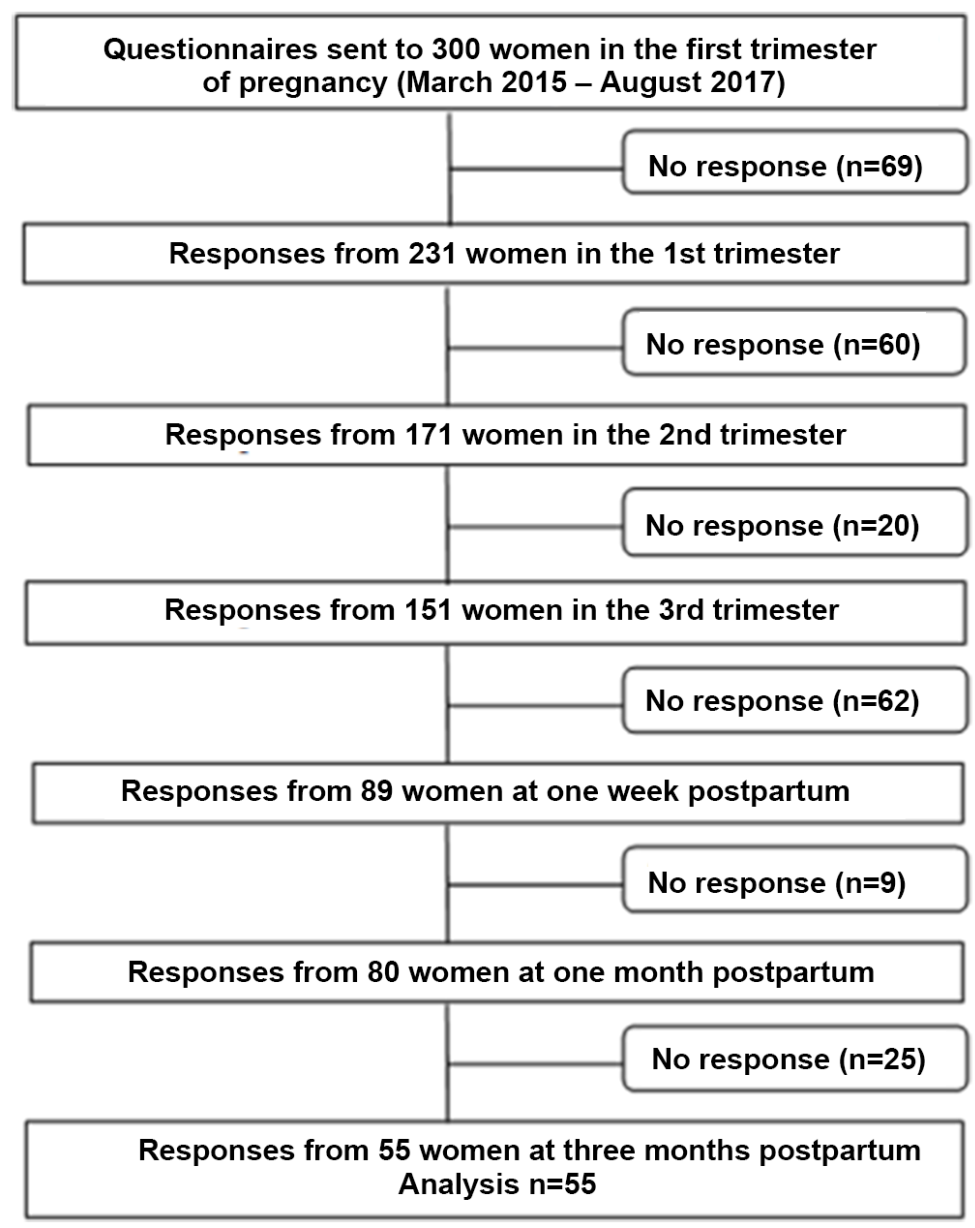

Figure 1. Recruitment of subjects.

\subsection{Questionnaire}

We designed a self-administered questionnaire consisting of three parts that required about 20 minutes to complete. The contents of the questionnaire were shown in detail in our previous report [5]. Briefly, the first part of the questionnaire consisted of questions regarding baseline characteristics such as age, marital status, education and weeks of pregnancy. The second part of the questionnaire consisted of questions regarding the presence of LBPP, location of pain, and $10 \mathrm{~cm}$ visual analog scale $(\mathrm{VAS})$ with end-points of no pain $(0 \mathrm{~cm})$ and worst thinkable pain $(10 \mathrm{~cm})$. We also asked about pregnancy mobility index (PMI) [13]. PMI has been developed to assess the ability to do normal household activities on a scale from "no problems performing this task" to "performing this task is impossible or only possible with the aid of others". PMI was shown to be a valid assessment of mobility during and after pregnancy [14]. Maternal mental health problems during pregnancy and in the postpartum period were assessed by using the Edinburgh Postnatal Depression Scale (EPDS). The EPDS questionnaire is a self-reported questionnaire consisting of ten items scored on a four-point Likert scale $(0-3)$ that is designed to assess pregnancy and postpartum depression [15]. The EPDS Japanese version was shown to have good inter- 
nal consistency (Cronbach alpha $=0.78$ ) and test-retest reliability (Spearman correlation $=0.92$ ) [16]. A score of more than 9 was designated to screen for minor and major depressive episodes, with sensitivities of $75 \%$ and $82 \%$ and with specificities of $93 \%$ and $95 \%$, respectively [16] [17].

\subsection{Data Analysis}

Cochran's Q test was used for comparison of changes in the proportions of women with LBPP in the 6 periods during pregnancy and in the postpartum period. Continuous VAS, PMI and EPDS scores in the 6 periods were compared by using Friedman test. The correlation of VAS score in one period with that in another period and the correlations of VAS score with PMI and EPDS score were analyzed by using Spearman's correlation. We compared the number of women with LBPP in one period and the number of women with LBPP in another period by using the chi-square test and Fisher's exact test. We classified the subjects by the VAS level at the first trimester into three groups; low group in which VAS level was less than the 25th percentile $(\mathrm{n}=20)$, moderate group ( $\mathrm{n}$ $=21$ ), and high group in which VAS level was more than the 75th percentile ( $\mathrm{n}=$ 14). Then, VAS, PMI, and EPDS scores between the three groups were compared by Median test. The proportions of women with menstrual pain, lumbar pain before pregnancy and a past history of LBPP in the previous pregnancy in the three groups of VAS at early pregnancy were compared by using Fisher's exact test. We performed logistic regression analysis with LBPP at 3 months postpartum as dependent variables and age, BMI, body weight of newborn infant, menstrual pain, lumbar pain before pregnancy as in-dependent variables. In addition, in multiparous women, we performed logistic regression analysis with LBPP at 3 months postpartum as dependent variables and age, BMI, body weight of newborn infant, menstrual pain, lumbar pain before pregnancy and past history of LBPP in the previous pregnancy as independent variables. $\mathrm{P}$ values were two-tailed, and those less than 0.05 were considered to be statistically significant. Statistical analyses of the data were carried out using SPSS version 24 for Windows (IBM Corp., Aromonk, NY).

\section{Results}

Baseline characteristics of the subjects are shown in Table 1. Mean age \pm standard deviation of the subjects was $32.3 \pm 4.9$ years. The subjects included $41.8 \%$ primiparous women and 58.2\% multiparous women. The proportion of women with a past history of LBPP in the previous pregnancy was $59.4 \%$.

\subsection{LBPP from the First Trimester until 3 Months Postpartum}

The proportions of women who complained of LBPP were $63.6 \%$ at the first trimester, $78.2 \%$ at the second trimester, $83.6 \%$ at the third trimester, $80.0 \%$ at one week postpartum, $69.1 \%$ at one month postpartum and $56.4 \%$ at 3 months postpartum. There was a significant difference in the proportion of women with 
Table 1. Characteristics of the subjects $(n=55)$.

\begin{tabular}{ccc}
\hline Age (years) & & $32.3 \pm 4.9$ \\
Parity & Primiparous & $23(41.8)$ \\
& Multiparous & $32(58.2)$ \\
Working (first trimester) & Yes & $44(80.0)$ \\
Presence of LBPP before pregnancy & No & $11(20.0)$ \\
& Yes & $25(45.5)$ \\
Past history of LBPP in previous pregnancy $(\mathrm{n}=32)$ & No & $30(54.5)$ \\
& Yes & $19(59.4)$ \\
\hline
\end{tabular}

The numbers in parenthesis indicate the proportion. Age is shown as mean \pm standard deviation. LBPP: low back and pelvic pain.

LBPP among the 6 stages $(\mathrm{p}=0.001)$ (Table 2). The proportion of women who had LBPP in all stages was $38.2 \%$, and the proportion of women who did not have LBPP in any of the stages was $5.4 \%$.

As shown in Table 2, there were significant differences in VAS scores among the 6 stages $(\mathrm{p}<0.001)$. VAS score at 3 months postpartum was significantly correlated with VAS scores at the first trimester $(r=0.410, p=0.002)$, second trimester $(r=0.298, p=0.027)$ and one month postpartum $(r=0.476, p<0.001)$ (Table 3).

\subsection{Associations of VAS Levels with PMI and EPDS}

PMI scores in the 6 stages are shown in Table 2. PMI score at 3 months postpartum was significantly correlated with PMI scores at the first trimester $(\mathrm{r}=$ $0.415, \mathrm{p}=0.001)$ and at one month postpartum $(\mathrm{r}=0.511, \mathrm{p}<0.001)$. Significant correlations of between VAS score and PMI were found at the first trimester $(\mathrm{r}=$ $0.773, \mathrm{p}<0.001)$, second trimester $(\mathrm{r}=0.661, \mathrm{p}<0.001)$, third trimester $(\mathrm{r}=$ $0.651, \mathrm{p}<0.001)$, one week postpartum $(\mathrm{r}=0.781, \mathrm{p}<0.001)$, one month postpartum $(\mathrm{r}=0.662, \mathrm{p}<0.001)$ and 3 months postpartum $(\mathrm{r}=0.766, \mathrm{p}<0.001)$. VAS score in each stage was 1.7 at the first trimester, 2.6 at the second trimester, 4.1 at the third trimester, 2.8 at one week postpartum, 2.2 at one month postpartum and 1.1 at 3 months postpartum (Table 2). VAS score at 3 months postpartum was significantly correlated with PMI scores at the first trimester $(\mathrm{r}=$ $0.428, \mathrm{p}=0.001)$ and at one month postpartum $(\mathrm{r}=0.353, \mathrm{p}=0.008)($ Table 3$)$. There were significant correlations between EPDS score at the first trimester and VAS score at the second trimester $(r=0.356, p=0.008)$, between EPDS score at the second trimester and VAS score at the third trimester $(r=0.391, p=0.003)$ and between EPDS score at the third trimester and VAS score at one week postpartum $(\mathrm{r}=0.292, \mathrm{p}=0.030)$. VAS score at 3 months postpartum was not associated with EPDS score in any of the stages (Table 3 ). 
Table 2. Changes in the proportions of women with LBPP and changes in LBPP, VAS, PMI and EPDS from pregnancy to the postpartum period.

\begin{tabular}{cccccccc}
\hline & 1st trimester & 2nd trimester & 3rd trimester & $\begin{array}{c}1 \text { week } \\
\text { postpartum }\end{array}$ & $\begin{array}{c}1 \text { month } \\
\text { postpartum }\end{array}$ & $\begin{array}{c}3 \text { months } \\
\text { postpartum }\end{array}$ & p-value \\
\hline $\begin{array}{c}\text { Gestational week } \\
\text { Proportion of women } \\
\text { with LBPP (\%) }\end{array}$ & $14.8(10-19)$ & $25.8(25-28)$ & $35.7(35-38)$ & & & & \\
VAS score & 63.6 & 78.2 & 83.6 & 80.0 & 69.1 & 56.4 & 0.001 \\
PMI & $1.7(0.0-4.9)$ & $2.6(0.6-5.6)$ & $4.1(2.7-6.1)$ & $2.8(1.1-5.1)$ & $2.2(0.0-4.2)$ & $1.1(0.0-3.3)$ & $<0.001$ \\
EPDS & $2.0(0.0-8.0)$ & $9.0(0.0-14.0)$ & $16.0(8.0-26.0)$ & $9.0(1.0-22.0)$ & $1.0(0.0-6.0)$ & $0.0(0.0-2.0)$ & $<0.001$ \\
& $5.0(4.0-8.0)$ & $4.0(2.0-7.0)$ & $4.0(2.0-7.0)$ & $6.0(1.0-10.0)$ & $5.0(3.0-10.0)$ & $3.0(1.0-6.0)$ & 0.001 \\
\hline
\end{tabular}

Gestational week is shown as mean (range). VAS, PMI and EPDS score are shown as medians. The numbers in parenthesis indicate the 25 - 75 percentiles. VAS: visual analog scale, PMI: Pregnancy Mobility Index, EPDS: Edinburgh Postnatal Depression Scale.

Table 3. Correlations of VAS at 3 months postpartum with VAS, PMI and EPDS at other stages.

\begin{tabular}{ccc}
\hline & Stage & VAS at 3 months postpartum \\
\hline 1st trimester & $0.410^{* *}$ \\
2nd trimester & $0.298^{*}$ \\
3rd trimester & 0.146 \\
1 week postpartum & -0.028 \\
1 month postpartum & $0.476^{* *}$ \\
1st trimester & $0.428^{* *}$ \\
2nd trimester & 0.198 \\
3rd trimester & 0.084 \\
1 week postpartum & 0.099 \\
1 month postpartum & $0.353^{* *}$ \\
3 months postpartum & $0.766^{* *}$ \\
\hline 1st trimester & -0.027 \\
2nd trimester & -0.146 \\
3rd trimester & -0.132 \\
1 week postpartum & -0.222 \\
1 month postpartum & -0.219 \\
3 months postpartum & -0.179 \\
\hline
\end{tabular}

${ }^{*} \mathrm{p}<0.05,{ }^{* *} \mathrm{p}<0.01$. VAS: Visual Analog Scale, PMI: Pregnancy Mobility Index. EPDS: Edinburgh Postnatal Depression Scale.

\subsection{Characteristics of Women with High VAS Score at Early Pregnancy}

As shown in Table 4, among the women with a high VAS score at early pregnancy, there was a significantly large proportion of women who had LBPP in a previous pregnancy $(\mathrm{p}=0.004)$. A high VAS score at early pregnancy was significantly associated with high VAS scores at the second trimester $(\mathrm{p}=0.034)$, at 
Table 4. Comparisons of the parameters according to the tertile of VAS scores at the first trimester.

\begin{tabular}{|c|c|c|c|c|c|}
\hline & \multicolumn{3}{|c|}{ VAS at the first trimester } & \multirow[t]{2}{*}{$\mathrm{p}$-value } \\
\hline & & Low & Moderate & High & \\
\hline & & $\mathrm{n}=20$ & $\mathrm{n}=21$ & $\mathrm{n}=14$ & \\
\hline VAS score & 1 st trimester & 0.0 & 1.9 & 6.4 & $<0.001$ \\
\hline Age (years) & & 33.5 & 32.2 & 30.7 & 0.406 \\
\hline \multirow{2}{*}{ Parity } & Primiparous & 8 & 12 & 3 & \multirow{2}{*}{0.100} \\
\hline & Multiparous & 12 & 9 & 11 & \\
\hline \multirow{2}{*}{ Menstrual pain } & Yes & 13 & 16 & 13 & \multirow{2}{*}{0.207} \\
\hline & No & 7 & 5 & 1 & \\
\hline \multirow{2}{*}{$\begin{array}{c}\text { Presence of LBPP before } \\
\text { pregnancy }\end{array}$} & Yes & 6 & 9 & 10 & \multirow{2}{*}{0.059} \\
\hline & No & 14 & 12 & 4 & \\
\hline \multirow{2}{*}{$\begin{array}{l}\text { Past history of LBPP in } \\
\text { previous pregnancy }(\mathrm{n}=32)\end{array}$} & Yes & 3 & 6 & 10 & \multirow{2}{*}{0.004} \\
\hline & No & 9 & 3 & 1 & \\
\hline \multirow[t]{5}{*}{ VAS score } & 2nd trimester & 0.7 & 2.2 & 4.9 & 0.034 \\
\hline & 3rd trimester & 3.5 & 3.7 & 5.1 & 0.151 \\
\hline & one week postpartum & 1.3 & 2.8 & 5.1 & 0.020 \\
\hline & one month postpartum & 0.0 & 3.0 & 3.2 & 0.005 \\
\hline & three months postpartum & 0.0 & 2.7 & 1.6 & 0.005 \\
\hline \multirow[t]{6}{*}{ PMI } & 1st trimester & 0.0 & 6.0 & 7.5 & $<0.001$ \\
\hline & 2nd trimester & 2.0 & 11.0 & 13.0 & 0.093 \\
\hline & 3rd trimester & 15.0 & 14.0 & 18.5 & 0.706 \\
\hline & one week postpartum & 6.0 & 13.0 & 9.5 & 0.120 \\
\hline & one month postpartum & 0.0 & 2.0 & 4.5 & 0.042 \\
\hline & three months postpartum & 0.0 & 2.0 & 0.0 & 0.019 \\
\hline \multirow[t]{6}{*}{ EPDS } & 1st trimester & 5.0 & 5.0 & 8.0 & 0.373 \\
\hline & 2nd trimester & 3.0 & 4.0 & 5.5 & 0.495 \\
\hline & 3rd trimester & 5.0 & 4.0 & 5.0 & 0.436 \\
\hline & one week postpartum & 6.5 & 6.0 & 6.0 & 0.985 \\
\hline & one month postpartum & 8.0 & 5.0 & 3.0 & 0.373 \\
\hline & three months postpartum & 2.0 & 3.0 & 4.0 & 0.959 \\
\hline
\end{tabular}

VAS, PMI and EPDS score are shown as medians. VAS: Visual Analog Scale, PMI: Pregnancy Mobility Index, EPDS: Edinburgh Postnatal Depression Scale.

1 week postpartum $(\mathrm{p}=0.020)$ and at 1 month postpartum $(\mathrm{p}=0.005)$. A moderate VAS score was significantly associated with a high VAS score at 3 months postpartum $(\mathrm{p}=0.005)$. A high VAS score at early pregnancy was significantly associated with high PMI scores at the first trimester $(\mathrm{p}<0.001)$ and 1 month postpartum ( $\mathrm{p}=0.042)$ but not with high PMI score at the second trimester, third trimester or 1 week postpartum. The VAS score at early pregnancy was not associated with EPDS levels during pregnancy and puerperium. 


\subsection{Predictive Factors for LBPP at 3 Months Postpartum}

As can be seen in Table 5, the presence of LBPP at 3 months postpartum was significantly associated with the proportions of women with LBPP at the first trimester, the second trimester, at one week postpartum and at one month postpartum but not with the proportion of women with LBPP at the third trimester. With respect to predictive factors for LBPP at 3 months postpartum, a past history of LBPP in the previous pregnancy was $7.68(1.15-50.98)(p=0.035)$ in multiparous women (Table 6). However, there was no predictive factor for LBPP at 3 months postpartum in all women.

\section{Discussion}

There was a large proportion of women with LBPP in the third trimester and $56.4 \%$ of the women had LBPP at 3 months postpartum. Olsson et al. [6] reported that the proportions of women with lumbopelvic pain were $44 \%$ at the second trimester and $38 \%$ at 6 months postpartum. Suzanne et al. [4] reported that the proportions of women with pelvic girdle pain were $73 \%$ at 30 weeks of pregnancy, $47.6 \%$ at 6 weeks postpartum and $42.7 \%$ at 12 weeks postpartum. Ostgaard et al. [1] reported that the proportions of women with low back pain were $44.6 \%$ at 25 weeks of pregnancy and $45.6 \%$ at 11 weeks postpartum. Thorell et al. [3] reported that the prevalence rates of lumbosacral and sacral pain were $30 \%-37 \%$ during pregnancy and significantly decreased to $11 \%-24 \%$ in the postpartum period. However, to our knowledge, there has been no prospective study on the proportions of women with LBPP during early pregnancy and at 3 months postpartum in Japan. In the present study, the proportion of women with LBPP at 3 months postpartum is high compared with the results of those

Table 5. Comparison of the number of women with LBPP and the number of women without LBPP between LBPP at 3 months postpartum and LBPP in each period during pregnancy and in the postpartum period.

\begin{tabular}{ccccc}
\hline & & LBPP of 3 months postpartum & \multirow{2}{*}{ p-value } \\
\cline { 3 - 4 } & & yes & no & \\
\hline LBPP of 1st trimester & yes & 27 & 8 & \\
& no & 4 & 16 & $<0.001$ \\
LBPP of 2nd trimester & yes & 28 & 15 & 0.016 \\
LBPP of 3rd trimester & no & 3 & 9 & 0.335 \\
LBPP of 1 week postpartum & yes & 27 & 19 & \\
LBPP of 1 month postpartum & no & 4 & 5 & 0.033 \\
& no & 3 & 16 & \\
& yes & 27 & 8 & 0.001 \\
\hline
\end{tabular}


Table 6. Logistic regression analysis with LBPP at 3 months postpartum.

\begin{tabular}{cccc}
\hline All women & & & $(\mathrm{n}=55)$ \\
\cline { 2 - 4 } Age & odds ratio & $95 \%$ CI & p-value \\
Body mass index & 1.02 & $0.90-1.14$ & 0.751 \\
Body weight of newborn infant & 1.02 & $0.87-1.20$ & 0.773 \\
Menstrual pain & 1.00 & $0.99-1.00$ & 0.958 \\
Presence of LBPP before pregnacy & 0.99 & $0.26-3.73$ & 0.990 \\
Multiparous women & 2.33 & $0.71-7.63$ & 0.160 \\
\hline Age & & & $(\mathrm{n}=32)$ \\
\cline { 2 - 4 } Body mass index & odds ratio & $95 \% \mathrm{CI}$ & $\mathrm{p}$-value \\
\hline Body weight of newborn infant & 1.11 & $0.78-1.57$ & 0.557 \\
Menstrual pain & 1.04 & $0.86-1.25$ & 0.658 \\
Presence of LBPP before pregnacy & 1.00 & $0.99-1.00$ & 0.403 \\
Past history of LBPP in previous pregnancy & 1.20 & $0.15-9.53$ & 0.862 \\
\hline
\end{tabular}

CI: Confidence interval, LBPP: Low back and pelvic pain.

previous studies. Also, 38\% of the women had LBPP continuously from early pregnancy until 3 months postpartum. The subjects who had LBPP at early pregnancy were interested in LBPP and they might be included in the higher proportion of women who had LBPP continuously.

Thorell et al. [3] reported that the intensity of back pain increased with advance of gestational age to a maximum level in late pregnancy and subsequently declined until 22 weeks postpartum. Our results are consistent with the results of that study. Also, women with a high intensity of LBPP at 3 months postpartum had a high intensity of LBPP at early pregnancy, suggesting that VAS level at early pregnancy is useful for predicting VAS level at 3 months postpartum. However, LBPP and VAS levels at the first trimester and 3 months postpartum were not associated with those at the third trimester, in which the proportion of women with LBPP and VAS level were the highest among the 6 stages. The factors responsible for occurrence of LBPP between early pregnancy and 3 months postpartum may be different from those at the third trimester. Morino et al. [18] reported that the activity of standing up from a chair was highly related to low back pain at early pregnancy and that the activity of tossing and turning was highly related to low back pain at late pregnancy. LBPP in late pregnancy may be involved in not only daily life activity but also change in posture accompanied by abdominal enlargement.

It has been reported that PMI was highest in late pregnancy and that PMI in women with low back pain was significantly higher than PMI in women without low back pain at early pregnancy, late pregnancy and 1 year postpartum [13]. 
We previously reported that women with a high intensity of VAS had difficulty for daily life activity during the second trimester and 1 month postpartum [8]. In the present study, a relationship between intensity of VAS and PMI as difficulty for daily life activity at 3 months postpartum was confirmed. Women with LBPP cannot perform motions for infant rearing such as holding a child in their arms and putting a child to bed. Physical burdens such as movement while holding a child increase because of the increase in body weight of the infant. Grant et al. [19] reported that women with back pain were likely to feel difficulties with parenting. Relief of LBPP leads to reduction of the difficulty for infant rearing thereafter. Since we showed that intensity of LBPP at 3 months postpartum was associated with difficulty of daily life activity at early pregnancy, assessment of PMI at early pregnancy may be useful for prediction for VAS at 3 months postpartum.

Mori et al. [20] reported that women whose EPDS was more than 9 were likely to have physical symptoms including back pain from the time of delivery to 6 months postpartum. After delivery, occurrence of various physical symptoms including LBPP may affect mental health. In the present study, the intensity of LBPP at 3 months postpartum was not associated with mental health. One reason for this is that the intensity of LBPP was lowest at 3 months postpartum. Iwata et al. [21] reported that factors such as concerns about infant caretaking and financial burden as well as physical burden in daily life were predictive factors for depression at 1 month postpartum. Thus, factors other than LBPP at 3 months postpartum might be associated with EPDS score.

Women who had a high intensity of LBPP at early pregnancy showed high intensity of LBPP until 1 month postpartum and had difficulty of daily life activity at 1 month postpartum. Morino et al. [18] reported that elementary motions that are a part of basic activities of daily life were related to LBPP in pregnant women. Women who had a high intensity of LBPP at early pregnancy may have motions that lead to LBPP in basic actions in daily life. In health guidance for women in early pregnancy, medical staff should provide information to women on body movements in daily life that can cause LBPP.

We showed that moderate VAS, but not high VAS, at early pregnancy was related to high intensity of LBPP and high PMI at 3 months postpartum. Many women spend much of their time resting until their 1 month postpartum checkup, but their physical burden increases in daily activity thereafter since the scope of activity increases after 1 month postpartum. Also, the physical burden of nursing, due to the increase in body weight of the infant, increases in postpartum women. Thus, LBPP in women whose VAS levels were moderate at early pregnancy might worsen since they did not initially feel severe pain. Also, no significant difference in the third trimester is due to high levels of VAS and PMI in the third trimester. A high VAS level at early pregnancy was not associated with EPDS score at any of the stages. Chang et al. [22] reported that pain at 3-5 days postpartum was associated with depression at 3 months postpartum. Other confounding factors may be related to the association of VAS with EPDS score. 
Only the VAS level at early pregnancy cannot be used to assess EPDS thereafter, and assessment of EPDS in each stage is needed.

Gutke et al. [2] found in a cohort study that identification of women with a risk for persistent pain at 3 months postpartum seems to be possible in early pregnancy. In the present study, we showed that VAS score at 3 months postpartum was correlated with VAS score at the first trimester and that the presence of LBPP at 3 months postpartum was significantly associated with the proportion of women with LBPP at the first trimester. Thus, LBPP at 3 months postpartum was associated with LBPP at early pregnancy. Robinson et al. [23] reported that the proportion of LBPP at 3 months postpartum did not change at one year postpartum. Wuytack et al. [24] reported that primiparous women who had continued pelvic girdle pain at 3 months postpartum had concerns about having another baby. Primiparous women with continuation of LBPP may decide not to have another pregnancy. Thus, reduction of LBPP at 3 months postpartum is important. It is necessary to manage LBPP at early pregnancy for prevention of LBPP at 3 month postpartum.

The predictive factor for LBPP at 3 months postpartum was a past history of LBPP before pregnancy in multiparous women. The result was in line with the result of previous study [12]. A past history of LBPP before pregnancy is a risk factor for occurrence of LBPP during pregnancy and LBPP may continue to postpartum period in multiparous women. In health guidance at early pregnancy, medical staff needs to assess LBPP and confirm a past history of LBPP before pregnancy. Care for LBPP at early pregnancy is important for both women with LBPP at early pregnancy and women with a past history of LBPP before pregnancy. Close et al. [25] suggested that health care professionals should initiate conversations about pregnancy-related LBPP in early pregnancy to educate women about the possibility of developing symptoms. A knowledge regarding the process of LBPP during pregnancy and postpartum period may be insufficient for primiparous women. It has also been reported that persistent symptoms were unexpected for primiparous women who had continued pelvic girdle pain at 3 months postpartum due to a lack of information about pain [24]. It is necessary to provide accurate information regarding LBPP at early pregnancy for primiparous women.

Care for LBPP at early pregnancy may result in improvement of postpartum LBPP. In Japan, health guidance for LBPP has been mainly done after the second trimester, in which the posture of pregnant women has changed. The results of the present study indicate that it is necessary to provide information about LBPP and assess LBPP at early pregnancy rather than at the second trimester. It is particularly important to provide information on LBPP at the stage of early pregnancy to women for whom there is a risk of LBPP occurring.

This study was a prospective study on LBPP from early pregnancy until 3 months postpartum. The study showed that assessment of LBPP at early pregnancy is important for preventing LBPP at 3 months postpartum and we have proposed the appropriate content of health guidance and the appropriate timing 
for such health guidance for pregnant women. However, this study has several limitations. First, compliance of response was low. Many of the women who dropped out were those at 1 week postpartum. The recovery rate might have been low because postmpartum women were busy for nursing. Second, the sample size was relatively small. Generalization of the results in our study may have a limitation. A significant association of LBPP with mental health may not have been found because of the small sample size.

In conclusion, the proportion of women with LBPP and the intensity of LBPP were increased at late pregnancy and gradually decreased until 3 months postpartum. LBPP at 3 months postpartum was associated with LBPP at the first trimester and a past history of LBPP in a previous pregnancy was an important factor for prediction of LBPP at 3 months postpartum. Provision of information regarding LBPP and confirmation of LBPP before pregnancy in addition to assessment of LBPP at early pregnancy are necessary for reduction of LBPP at 3 months postpartum.

\section{Acknowledgements}

The authors are grateful to doctors, midwives and medical assistants in Shikoku Medical Center for Children and Adults for supporting this research. We also thank the women who participated in this study.

\section{Ethics Approval and Consent to Participate}

The study protocol was reviewed and approved by the Ethics Review Board of Tokushima University Hospital (approval no. 2201). This study was reviewed by the Ethics Review Board of a birth center in Kagawa Prefecture in Japan.

\section{Conflicts of Interest}

The authors declare no conflicts of interest regarding the publication of this paper.

\section{References}

[1] Ostgaard, H.C., Roos-Hansson, E. and Zetherström, G. (1996) Regression of Back and Posterior Pelvic Pain after Pregnancy. Spine, 21, 2777-2780. https://doi.org/10.1097/00007632-199612010-00013

[2] Gutke, A., Ostgaard, H.C. and Oberg, B. (2008) Predicting Persistent Pregnancy-Related Low Back Pain. Spine, 33, E386-E393. https://doi.org/10.1097/BRS.0b013e31817331a4

[3] Thorell, E. and Kristiansson, P. (2012) Pregnancy Related Back Pain, Is It Related to Aerobic Fitness? A Longitudinal Cohort Study. BMC Pregnancy and Childbirth, 12, 30. https://doi.org/10.1186/1471-2393-12-30

[4] Stomp-van den Berg, S.G., Hendriksen, I.J., Bruinvels, D.J., Twisk, J.W., van Mechelen, W. and van Poppel, M.N. (2012) Predictors for Postpartum Pelvic Girdle Pain in Working Women: The Mom@Work Cohort Study. Pain, 153, 2370-2379. https://doi.org/10.1016/j.pain.2012.08.003

[5] Uemura, Y., Yasui, T., Horike, K., Maeda, K., Uemura, H., Haku, M., Sakae, R. and 
Matsumura, K. (2017) Factors Related with Low Back Pain and Pelvic Pain at the Early Stage of Pregnancy in Japanese Women. International Journal of Nursing and Midwifery, 9, 1-9. https://doi.org/10.5897/IJNM2016.0249

[6] Olsson, C.B., Nilsson-Wikmar, L. and Grooten, W.J. (2012) Determinants for Lum bopelvic Pain 6 Months Postpartum. Disability and Rehabilitation, 34, 416-422. https://doi.org/10.3109/09638288.2011.607212

[7] Bergström, C., Persson, M. and Mogren, I. (2016) Sick Leave and Healthcare Utilisa tion in Women Reporting Pregnancy Related Low Back Pain and/or Pelvic Girdle Pain at 14 Months Postpartum. Chiropractic and Manual Therapies, 24, 7.

[8] Uemura, Y., Yasui, T., Horike, K., Maeda, K., Uemura, H., Haku, M., Sakae, R. and Matsumura, K. (2017) Association of Low Back and Pelvic Pain at the Second Trimester with That at the Third Trimester and Puerperium in Japanese Pregnant Women. Journal of Pregnancy Child Health, 4, 5. https://doi.org/10.4172/2376-127x.1000351

[9] Perlen, S., Woolhouse, H., Gartland, D. and Brown, S.J. (2013) Maternal Depression and Physical Health Problems in Early Pregnancy: Findings of an Australian Nulliparous Pregnancy Cohort Study. Midwifery, 29, 233-239.

https://doi.org/10.1016/j.midw.2012.01.005

[10] Woolhouse, H., Gartland, D., Perlen, S., Donath, S. and Brown, S.J. (2014) Physical Health after Childbirth and Maternal Depression in the First 12 Months Postpartum: Results of an Australian Mulliparous Pregnancy Cohort Study. Midwifery, 30, 378-384. https://doi.org/10.1016/j.midw.2013.03.006

[11] Gutke, A., Josefsson, A. and Oberg, B. (2007) Pelvic Girdle Pain and Lumbar Pain in Relation to Postpartum Depressive Symptoms. Spine, 32, 1430-1436. https://doi.org/10.1097/BRS.0b013e318060a673

[12] Mukkannavar, P., Desai, B.R., Mohanty, U., Parvatikar, V., Karwa, D. and Daiwajna, S. (2013) Pelvic Girdle Pain after Childbirth: The Impact of Mode of Delivery. Journal of Back Musculoskeletal Rehabilitation, 26, 281-290. https://doi.org/10.3233/BMR-130378

[13] Van de Pol, G., de Leeuw, J.R., van Brummen, H.J., Bruinse, H.W., Heintz, A.P. and van der Vaart, C.H. (2006) The Pregnancy Mobility Index: A Mobility Scale during and after Pregnancy. Acta Obstetricia et Gynecologica, 85, 786-791. https://doi.org/10.1080/00016340500456373

[14] Sabino, J. and Grauer, J.N. (2008) Pregnancy and Low Back Pain. Current Reviews in Musculoskeletal Medicine, 1, 137-141. https://doi.org/10.1007/s12178-008-9021-8

[15] Cox, J.L., Holden, J.M. and Sagovsky, R. (1987) Detection of Postnatal Depression. Development of the 10-Item Edinburgh Postnatal Depression Scale. The British Journal of Psychiatry, 150, 782-786. https://doi.org/10.1192/bjp.150.6.782

[16] Okano, T., Murata, M., Masuji, F., Tamaki, R., Nomura, J., Miyaoka, H. and Kitamura, T. (1996) Validation and Reliability of Japanese Version of EPDS (Edinburgh Postnatal Depression Scale). Archives of Psychiatric Diagnosis and Clinical Evaluation, 74, 525-533.

[17] Yamashita, H., Yoshida, K., Nakano, H. and Tashiro, N. (2000) Postnatal Depression in Japanese Women. Detecting the Early Onset of Postnatal Depression by Closely Monitoring the Postpartum Mood. Journal of Affective Disorders, 58, 145-154. https://doi.org/10.1016/S0165-0327(99)00108-1

[18] Morino, S., Ishihara, M., Umezaki, F., Hatanaka, H., Iijima, H., Yamashita, M., Aoyama, T. and Takahashi, M. (2017) Low Back Pain and Causative Movements in 
Pregnancy: A Prospective Cohort Study. BMC Musculoskeletal Disorders, 18, 416.

[19] Grant, M.I., Foster, N.E., Wright, C.C., Barlow, J.H. and Cullen, L.A. (2004) Being a Parent or Grandparent with Back Pain, Ankylosing Spondylitis or Rheumatoid Arthritis: A Descriptive Postal Survey. Musculoskeletal Care, 2, 17-28. https://doi.org/10.1002/msc.53

[20] Mori, E., Iwata, H., Sakajo, A. and Maehara, K. (2017) Association between Physical and Depressive Symptoms during the First 6 Months Postpartum. International Journal of Nursing Practice, 23, e12545. https://doi.org/10.1111/ijn.12545

[21] Iwata, H., Mori, E., Tsuchiya, M., Sakajo, A., Maehara, K., Ozawa, H., Morita, A., Maekawa, T., Aoki, K. and Tamakoshi, K. (2016) Predictors of Depressive Symptoms in Older Japanese Primiparas at 1 Month Post-Partum: A Risk-Stratified Analysis. Japan Journal of Nursing Science, 13, 147-155.

https://doi.org/10.1111/jjns.12099

[22] Chang, S.R., Chen, K.H., Lee, C.N., Shyu, M.K., Lin, M.I. and Lin, W.A. (2016) Relationships between Perineal Pain and Postpartum Depressive Symptoms: A Prospective Cohort Study. International Journal of Nursing Studies, 59, 68-78. https://doi.org/10.1016/j.ijnurstu.2016.02.012

[23] Robinson, H.S., Vøllestad, N.K. and Veierød, M.B. (2014) Clinical Course of Pelvic Girdle Pain Postpartum. Manual Therapy, 19, 190-196. https://doi.org/10.1016/j.math.2014.01.004

[24] Wuytack, F., Curtis, E. and Begley, C. (2015) Experiences of First-Time Mothers with Persistent Pelvic Girdle Pain after Childbirth: Descriptive Qualitative Study. Physical Therapy, 95, 1354-1364. https://doi.org/10.2522/ptj.20150088

[25] Close, C., Sinclair, M., Liddle, D., McCullough, J. and Hughes, C. (2016) Women's Experience of Low Back and/or Pelvic Pain (LBPP) during Pregnancy. Midwifery, 37, 1-8. https://doi.org/10.1016/j.midw.2016.03.013 